\title{
Primary hip arthroplasty for the treatment of alkaptonuric hip arthritis: 3- to 24-year follow-ups
}

\author{
Javahir A. Pachore ${ }^{1}$, Vikram Indrajit Shah ${ }^{1,2}$, Sachin Upadhyay ${ }^{3,4^{*}} \mathbb{D}$, Kalpesh Shah ${ }^{5}$, Ashish Sheth $^{5}$ and \\ Amish Kshatriya ${ }^{5}$
}

\begin{abstract}
Background: The objective of this study is to share our experience in total hip replacement for the treatment of ochronotic hip arthritis, in particular to report how to establish the diagnosis and some tips to limit complications.

Method: A cohort comprised of 10 patients (12 hips) with alkaptonuric hip arthritis. There were six men and four women with the mean age of $62.80 \pm 7.57$ years. All patients had a stiff spine, grossly restricted movements of hip joints, and severely limited daily routine activities. Total hip replacement was performed in all patients. The patients were evaluated at 6, 12, and 24 months after surgery, as well as every 4 years thereafter. Harris hip score was used to assess the functional outcome. The level of significance was set at $p<0.05$.

Results: The mean follow-up lasted $16.70 \pm 6.82$ years ( 3 to 24 years). At the final available follow-up, nine patients returned to work, ambulate without an orthosis, and achieve complete pain relief. Harris hip score was improved from poor to excellent. One patient died 16 years after surgery due to breast cancer. No complication relating to prosthetic failures was detected.
\end{abstract}

Conclusion: Total hip replacement gives long-term satisfactory results in patients with alkaptonuric hip arthritis, resulting in comparable function of the hips in patients who undergo primary osteoarthrosis.

Keywords: Total hip replacement, Alkaptonuric arthritis, Ochronosis, Harris hip score

\section{Background}

First described by Virchow in 1866, ochronosis is the connective tissue manifestation of alkaptonuria, an autosomal recessive mutation of the $\mathrm{HGO}$ gene on chromosome $3 \mathrm{q}$, caused by deficiency of homogentisate 1,2 dioxygenase activity [1]. Homogentisic acid oxidase is responsible for turnover of homogentisic acid (HGA) during the course of phenylalanine and tyrosine catabolism [2]. HGA accumulates and is polymerized into a blue-black pigment that is ultimately deposited in skin, bones, tendons, articular cartilages, synovial membranes, lungs, valves, and kidneys [3]. The accumulation eventually causes severe degeneration of

\footnotetext{
* Correspondence: drsachinupadhyay@gmail.com

Institution(s) at which the work was performed: Shalby Hospitals in Ahmedabad, Gujarat

${ }^{3}$ Department of Orthopaedics, NSCB Medical College, Jabalpur, MP, India ${ }^{4}$ Department of Trauma, Joint Replacement and Minimal Invasive Surgery, Shalby Hospitals Jabalpur, Jabalpur, Madhya Pradesh, India Full list of author information is available at the end of the article
}

the spine and peripheral joints, which may clinically lead to common arthritic disorders [4]. Alkaptonuria is a rare metabolic disorder characterized by a triad of degenerative arthritis, ochronotic pigmentation, and homogentisic aciduria, affecting one in 250,000 to 1 million people $[5,6]$. Chromatographic, enzymatic or spectrophotometric determinations of HGA are confirmatory tests. Currently, there is no definitive cure for alkaptonuric ochronosis. Symptomatic treatment of the complications of alkaptonuria is the only option, including pain management, physiotherapy, chiropractic care, and instruction regarding a home exercise program. A successful treatment for tendon ruptures caused by ochronosis is primary repair [6]. High dose of vitamin $\mathrm{C}$ decreases urinary benzoquinone acetic acid, but has no effect on HGA excretion and, moreover, no credible studies have shown that treatment with vitamin $C$ is clinically effective. Nitisinone, a potent inhibitor of 4hydroxyphenylpyruvate dioxygenase, dramatically reduces

(C) The Author(s). 2019 Open Access This article is distributed under the terms of the Creative Commons Attribution 4.0 International License (http://creativecommons.org/licenses/by/4.0/), which permits unrestricted use, distribution, and 
production and urinary excretion of homogentisic acid [7], however, the effectiveness of Nitisinone in treating ochronosis remains unknown. Patients with alkaptonuria are usually asymptomatic, and the ochronotic arthropathy appears after the fourth decade [8]. Total replacement of hip, knee, elbow, and shoulder can alleviate pain and increase patient's daily activities [2]. There is a paucity of studies concerning total hip replacement in the present patient population with adequate follow-up statistics.

The primary objective of the present study is to share our experience in total hip replacement (THR) for ochronotic hip arthritis, in particular to report on establishment of diagnosis and tips to decrease the complications.

\section{Patients and methods}

The study was approved by the Institutional Ethics Committee of the hospital. Informed consent was obtained from each patient.

A case series of 10 patients (12 hips) with alkaptonuric hip arthritis who presented to our institute were reported. There were six men and four women with the mean age of $62.80 \pm 7.57$ years (range, $53-80$ years). Demographic variables, presentation, comorbidities, and preoperative diagnoses were recorded and analyzed (Table 1). All patients had pain in groin region for the past 4 months to several years. None of the patients was under specific treatments. Clinical, imaging, and laboratory assessments were performed in all patients. Of the 10 patients, seven patients had a family history of alkaptonuria, and eight patients had cutaneous signs of ochronosis. Nine patients had a history of blackish discoloration of urine on exposure to air. Spine examination revealed stiff spine with limited movements in all patients. On local examination, hip movements were painful and severely restricted. All patients reported gross limitation of daily routine activities and needed assisted ambulation. A dried urine spot (DUS) [9] detects HGA in all patients. Other laboratory parameters were within the normal ranges. The standard radiographs of hip showed severe joint degeneration and narrowing of the joint space with irregularity and flattening of the femoral head (Fig. 1a, b). A lateral spine radiograph revealed osteoporosis, flattened and intra-discal calcification, and variable degrees of fusion of the vertebral bodies (Fig. 2a, b). Pre-operatively, the mean Harris Hip Score was $35.00 \pm 14.06$. All patients were diagnosed with ochronotic hip arthritis, and underwent primary hip arthroplasty. Eight patients underwent unilateral surgery and two had bilateral procedures. In bilateral cases, the priority was given to the side with more severely affected joint.

\section{Surgical technique}

All surgeries were performed by the same senior surgeon and the same arthroplasty team through the posterior approach with the patients in the lateral decubitus position. The incision was made over the center of the trochanteric region and then was adequately lengthened both cranially and caudally for a good exposure to avoid forceful retraction. The subcutaneous fat was incised deep to the fascia lata. We found black discoloration over the tensor fascia lata, and calcification and hard tissue at the insertion of gluteus maximus tendon with blackish deposits (Fig. 3). We found deposition of black tissues with some degrees of fragility in the gluteus medius tendon. The fascia lata was incised and the gluteus maximus tendon was bluntly splited along the direction of fibers. The trochanteric bursa overlying the external rotators was incised. The sciatic nerve was carefully protected. The short external rotators were tagged with a suture for identification and subsequent repair. These rotators were detached at their insertions onto the greater trochanter and reflected posteriorly to expose the posterior joint capsule. The capsule was found to be more fibrotic and contracted with blackish discoloration. If the capsule was hard and contracted, we usually incised the capsule from 6 o'clock to 12 o'clock to ease dislocation of the femoral head. When the hip was dislocated, we found the femoral head was covered with typical black painted articular cartilage, but the subchondral bone was not affected (Fig. 4a). The tip of the trochanter was impregnated with thick black pigments. During the preparation of the femur, great care should be taken to avoid fracturing the tip of trochanter. Preparation of the acetabular bone was difficult due to the black deposition that looked like a layer of tissue (Fig. 4b). The bone was sclerotic, hard, and difficult to ream, so as to open subchondral bone (Fig. 4b). We conducted either pre-drilling or curettage of the cup and finally reamed it to get punctate bleeding. Furthermore, owing to the sclerotic rim cup, expansion of the acetabulum was probably difficult. Therefore, additional screws were used to secure the fixation. After trailing, the final cup position was achieved within the safe zone for both anteversion and inclination. The femoral preparation was done in the routine fashion, because the femoral canal was normal. After placement of the optimal stem and femoral head, THR was completed and hip joint was reduced. Closure was done in layers under negative suction drain.

\section{Postoperative managements}

The pigmented cartilaginous surface, bone, and soft tissues were sent for histomorphological evaluation. Our protocol was to remove the drain on the second postoperative day. The patient was discharged on the sixth postoperative day, and then given a standard rehabilitation.

\section{Postoperative evaluation}

All patients returned for follow-ups at 6, 12, and 24 months, as well as every 4 years thereafter. Preoperatively 


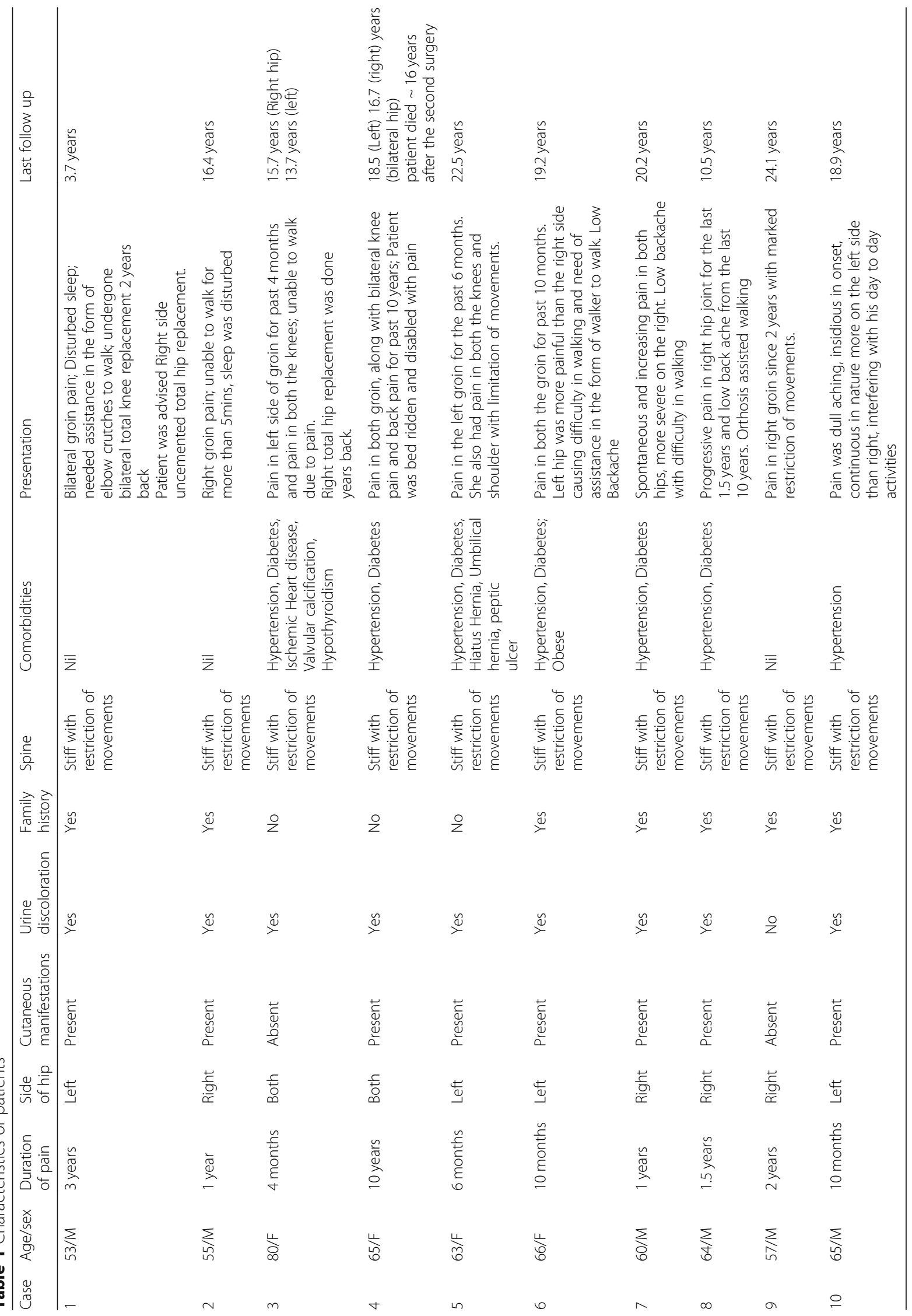




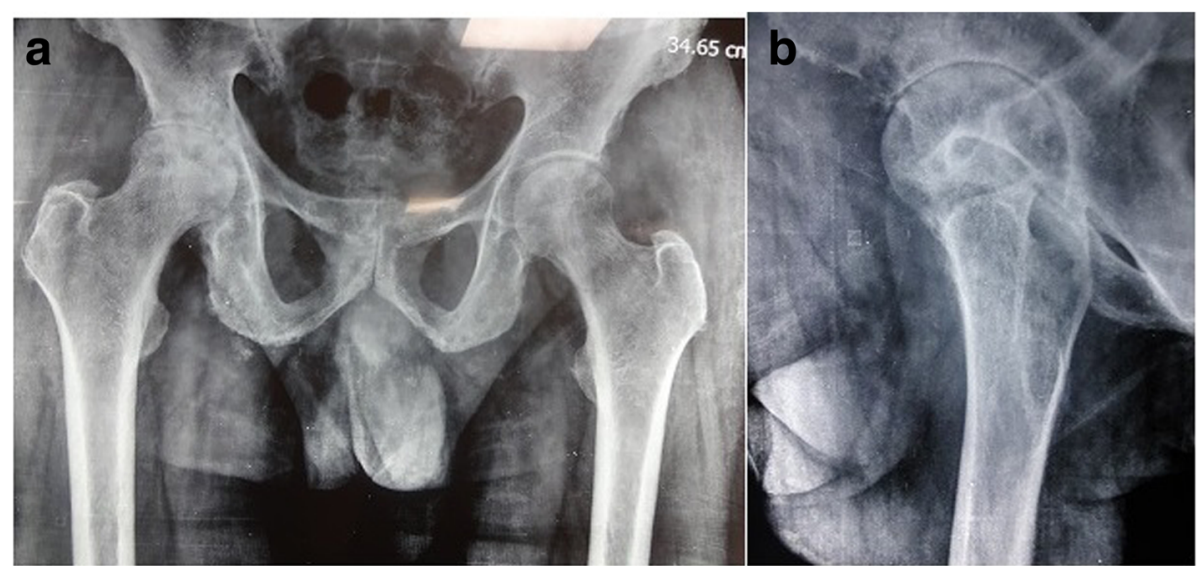

Fig. 1 a \& $\mathbf{b}$ Anteroposterior and lateral X-ray films of the pelvis, with both hip joints showing reduced join space with degenerative changes, irregularity, and flattening of femoral head

and at the final follow-up, functional outcome evaluation was performed using HHS. Standard anteroposterior and lateral femoral X-rays were obtained to study heterotopic ossification, radiolucent lines, position of stem and subsidence, the change in the cup inclination or cup migration, and loosening of implants. All parameters were expressed as mean \pm standard deviation. The differences were analyzed with student's $t$ test. A $p<0.05$ was considered statistically significant.

\section{Results}

In this series, the postoperative course was uneventful. No intraoperative complications other than increased blood loss were observed. Histopathological examination of periarticular tissues revealed that tissue pigmentation was most markedly in the deeper layers, with areas of calcification and degeneration. The synovium was thickened, inflamed, and pigmented. HGA was detected using dried urine spots [9] in all patients. In all cases,

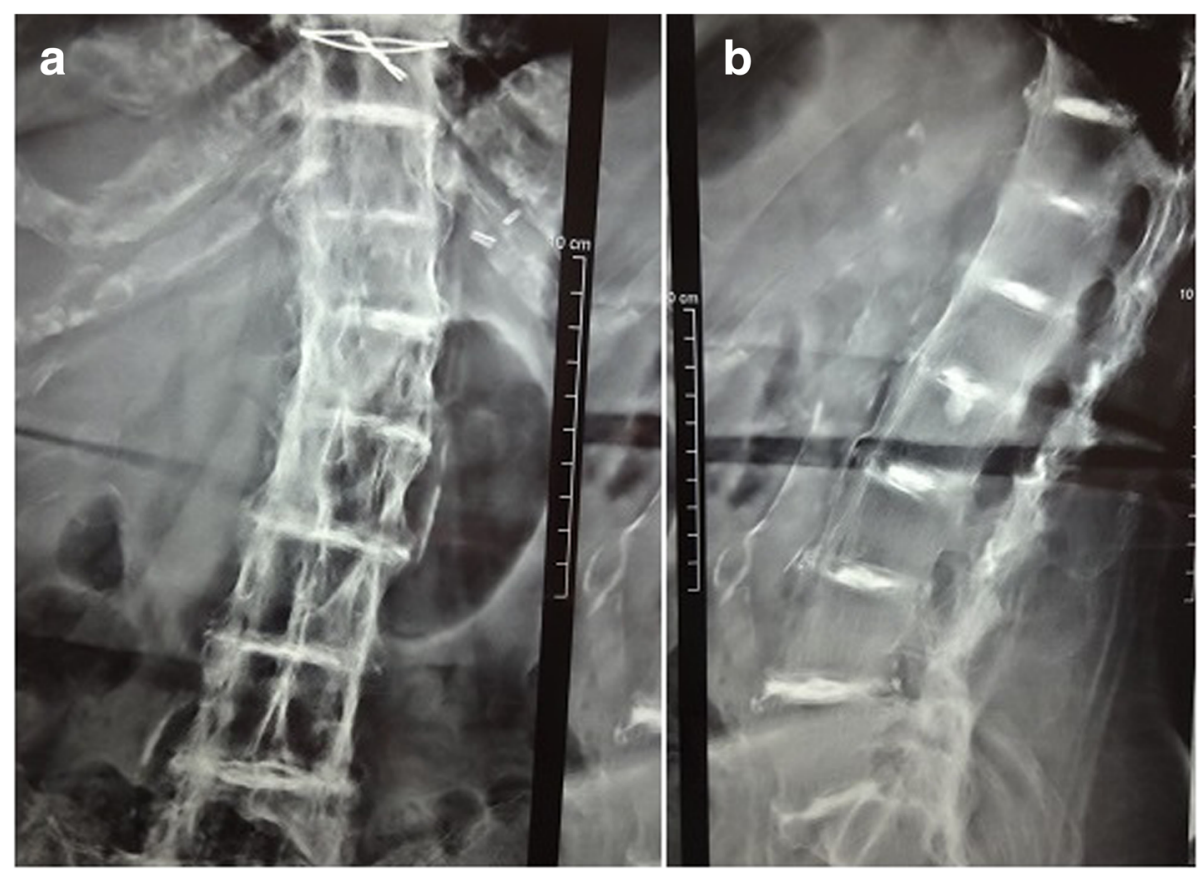

Fig. 2 a \& b Anteroposterior and lateral spine X-ray films revealed osteoporosis, flattened, and intra-discal calcification (doubling signs), and variable degrees of fusion of the vertebral bodies 


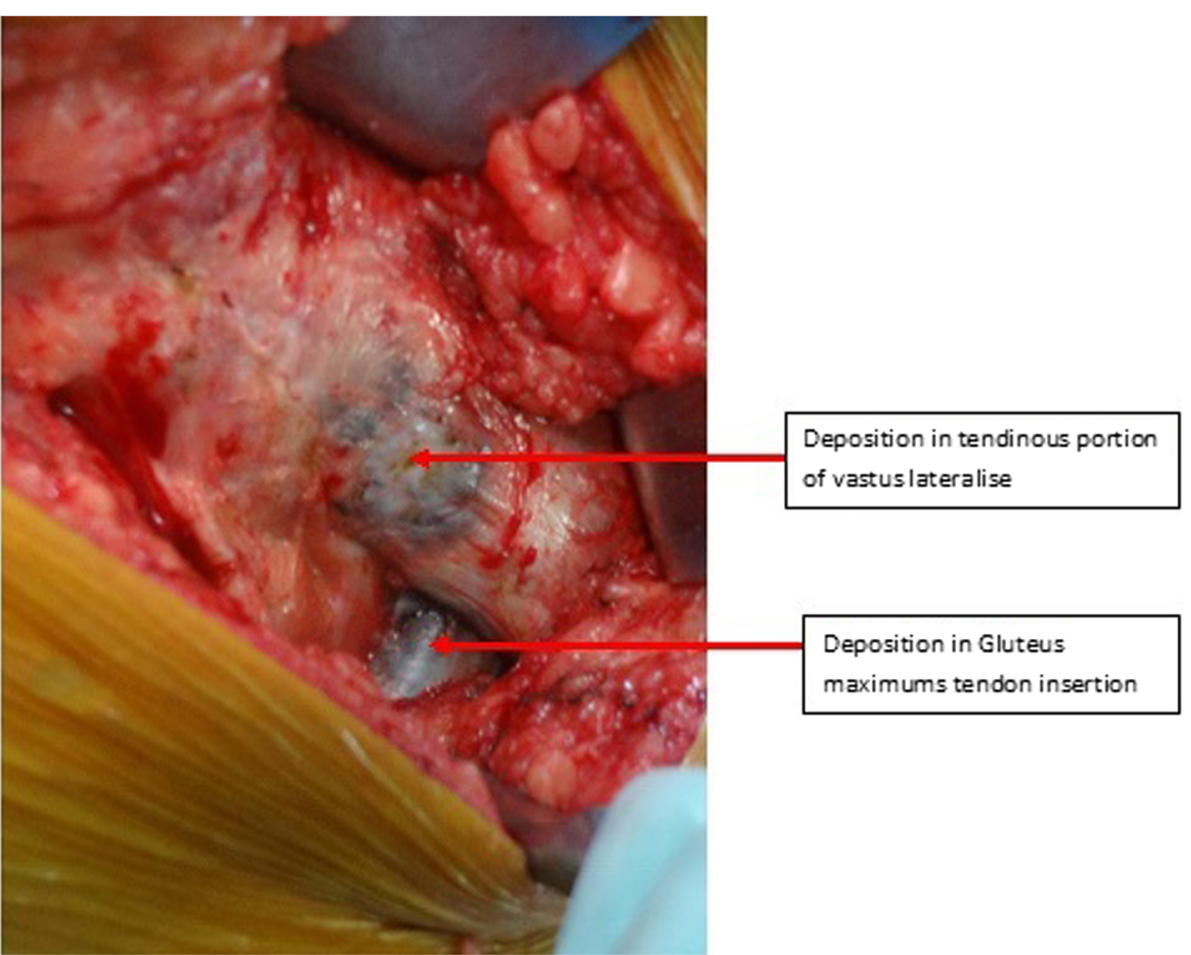

Fig. 3 Black discoloration over the tensor fascia lata, and calcification of the hard tissues with blackish deposit over the gluteus muscles

there was a macroscopic layer of black pigmented articular cartilage, which was more severe in elderly patients. Histopathological, physical, biochemical, and radiographic evaluations confirmed the diagnosis of ochronotic arthropathy. One patient died 16 years after the second hip surgery due to breast cancer. Therefore, a total of 10 hips were followed-up finally. The mean follow-up time was $16.70 \pm 6.82$ years (range, 3 to 24 years). At 6-month follow-up, the mean HHS improved significantly, from $35.00 \pm 14.06$ preoperatively to $93.20 \pm 1.75$ $(p<0.05)$. Radiographs showed stable prostheses in situ without any evidence of subsidence (Fig. 6a, b, c, d, e, f, g and Fig. 7a, b, c, d). The latest follow-up showed that nine patients (10 hips) went back to work and ambulated without walking aids. All patients had complete pain relief. HHS was improved from poor to excellent. Although patients had complaints pertaining to low backache, none of the patient had major complications requiring a revision surgery. We did not found complications related to prosthetic failures (Fig. 6a, b, c, d, e, f, $\mathrm{g}$ and Fig. 7a, b, c, d).

\section{Discussion}

Alkaptonuria is a rare autosomal recessive inborn metabolic disorder of tyrosine metabolism due to deficiency of homogentisic oxidase enzyme. Alkaptonuria is characterized by excretion of homogentisic acid in urine, and deposition of oxidized homogensitate pigments in the
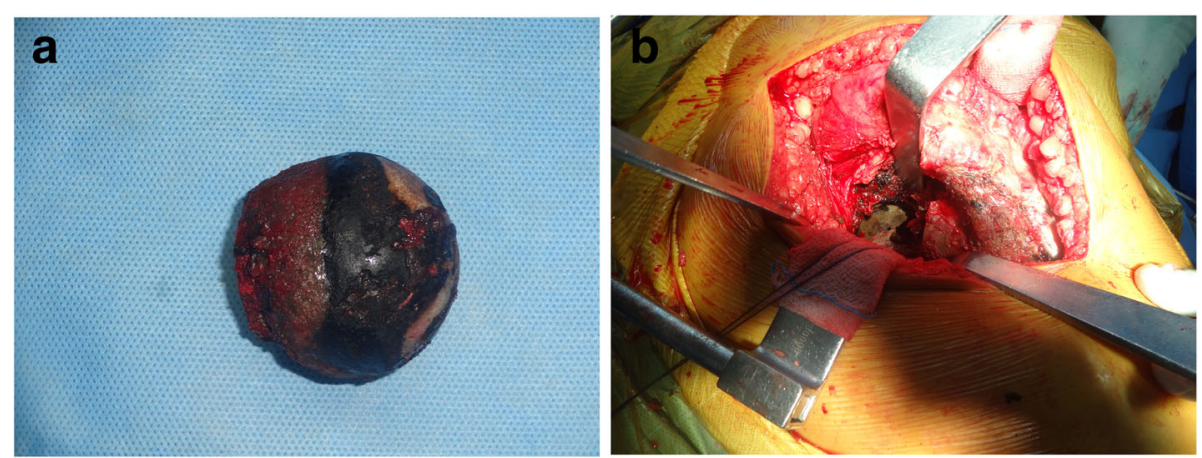

Fig. 4 a A classical black painted articular cartilage of the femoral head; b A black painted articular cartilage of the acetabulum with sclerosis 
connective tissues and articular cartilages (ochronosis). The gene for this pathological condition is present at locus 3q21-23 [10]. In ochronosis, there is deposition of pigments from homogentisic acid in all types of connective tissues, including cartilage, cardiovascular system, genitourinary system, sclera, skin, and ear cartilage [11, 12]. Urine discoloration is the first clinical manifestation of alkaptonuria, followed by color changes of the sclera and ears. Based on the detailed general examination, cutaneous signs of ochronosis include color changes of the sclera and ears which can easily be observed (Fig. 5). 80 $\%$ of cases in our series had positive association. There is a paucity of available literature concerning the occurrence of ochronosis arthropathy without ocular and cutaneous signs. Kusakabe et al. [13] showed cervical arthropathy without ocular and dermatological findings. Retrospective inquiry for the family history of alkaptonuria usually makes sense. In the present ten patients, seven had a positive family history. Patients will respond to the leading question about discoloration of urine. Ninety percent of the patients in our series had a history of blackish discoloration of urine on exposure to air.

The tendons and ligaments may also be affected because of their high collagen content. It causes inflammatory alterations resulting in rupture of the tissues [6]. Early diagnosis of ochronosis is valuable to avoid tendon ruptures. The preoperative cardiac clearance is imperative to rule out the risk of valvular calcification [12]. In our series, one patient who had a history of valvular calcification underwent aortic valve replacement and coronary artery bypass graft surgery.

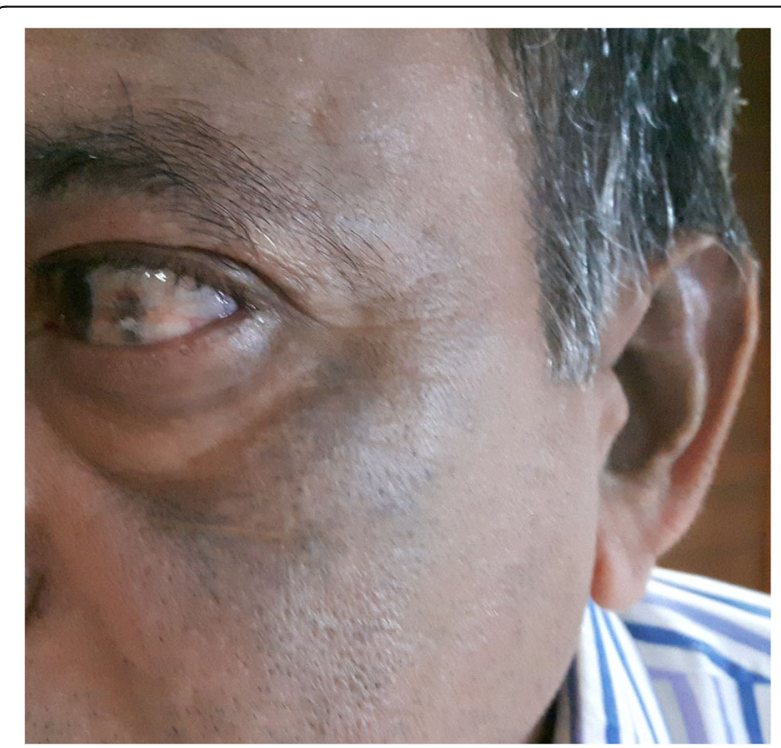

Fig. 5 Cutaneous signs of ochronosis that include changes of the color of the sclera and ears
Alkaptonuric arthropathy has previously been shown to be a relatively benign disease. Our evidence showed that the patients with this disease were crippled and disabled with pain. Recent overall increase in life expectancy may account for the scenario. Although alkaptonuria affects both men and women with ochronotic arthropathy, the trend is more severe and more frequent for men than for women. The result is also male preponderance (six men), which was compatible with our series [14, 15].

Patients with alkaptonuria are usually asymptomatic, and arthropathy appears after the third or fourth decade with a sudden onset of pain limiting daily routine activities $[15,16]$. A late onsets is attributed to the gradual age-related decline in the renal ability to excrete homogentisic acid, resulting in a diseased association with signs and symptoms of accumulation of homogentisic acid. In about $50 \%$ of patients, alkaptonuric cases develops arthropathy [17]. Low backache precedes joint diseases. In our cases, low backache with restricted mobility was on the foreground. In most of the cases, spinal examination showed the restricted movements of the spine. Back pain and stiffness in the thoraco-lumber junction and cervical region may be the initial symptom of ochronosis. The spinal stiffness is almost like ankylosing spondylitis but the age group is different. Pain is more severe in patients with ankylosing spondylitis than in patients with ochronosis, which have an increased degeneration that may not proportionally increase severity of pain. There is a loss of cervical or lumbar lordosis. There may be kyphosis or localized scoliosis. In a few of such cases, root pain or sciatica could be the presenting or only symptom in ochronotic arthropathy. Spine radiograph shows intra-discal calcifications giving a "doubling" of the outline, which confirmed the diagnosis of ochronosis [18]. In patients with ankylosing spondylitis, there is extensive ossification of spinal ligaments with little calcification of the intervertebral discs.

Ochronotic arthropathy usually involves the large weight-bearing joints (knee and hip joints) rather than the small joints of the hand and foot [19-21]. In our series, the hip joints were severely affected. Movements of the hip joints were painful and were restricted due to the arthropathies. Similar to patients with primary osteoarthrosis, concentric reduction of the joint space is frequently seen on X-rays. However, the radiological alterations may be much less apparent than the clinical manifestations.

Intraoperatively, we noticed an increased blood loss, which may be the result of en bloc synovectomy of the hypertrophied synovium. The blood loss was more than what is usually observed in arthritis of other common causes. Although the hemoglobin and haematocrit levels were not decreased after surgery, we still advise to avoid total synovectomy without intraoperative bleeding control. 


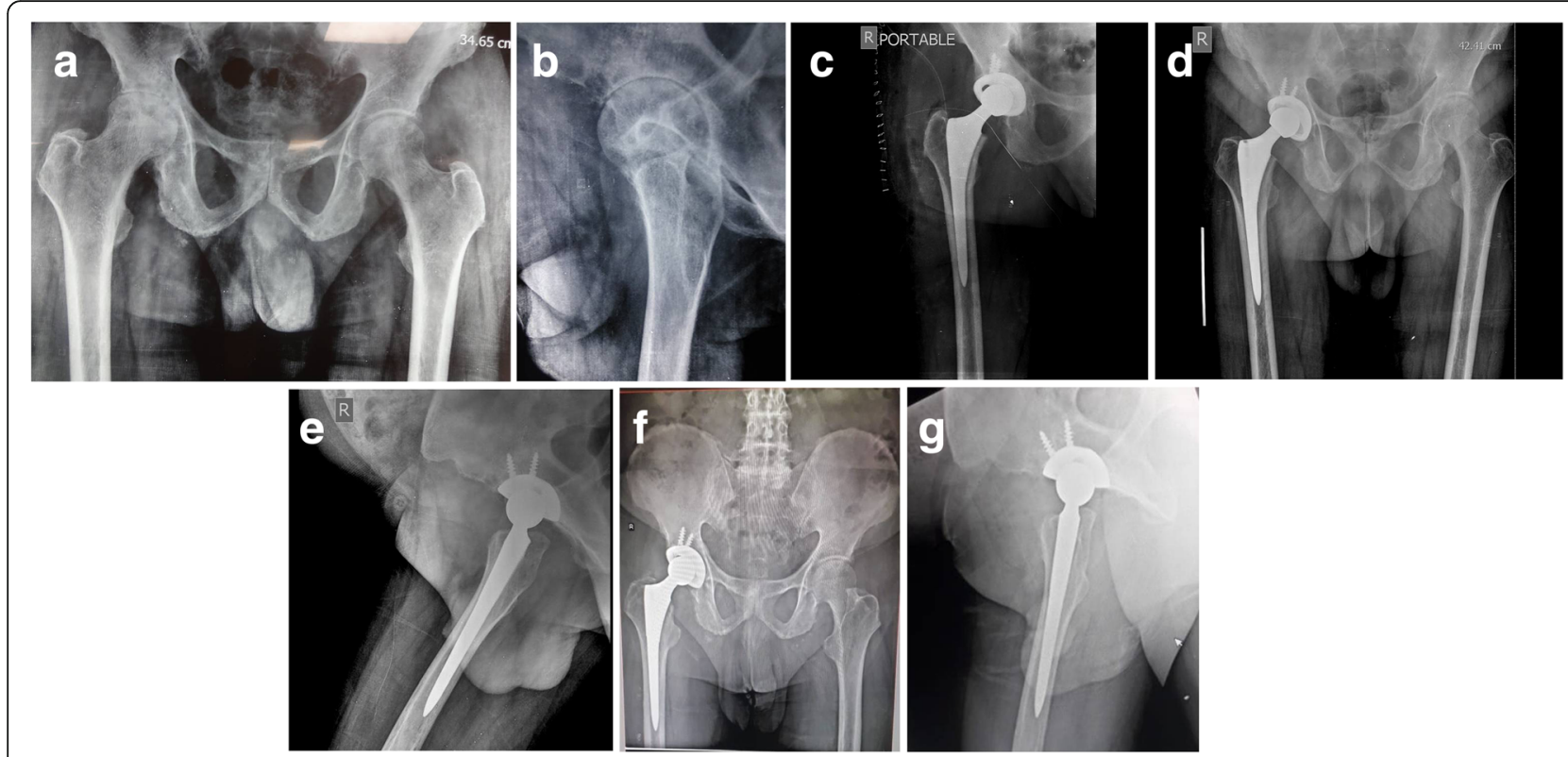

Fig. 6 Pelvis and both hips on X-rays. a preoperative anteroposterior view; b lateral view c anteroposterior view immediately after surgery; $\mathbf{d}$ anteroposterior view 5 years after surgery; e Lateral view 5 years after surgery; $\mathbf{f}$ anteroposterior view 10.5 years after surgery; $\mathbf{g}$ Lateral view 10.5 years after surgery

In ochronotic cases, Cebesoy et al. [22] has recommended complete removal of the joint capsule. We speculated that complete capsular resection could increase the rate of dislocation postoperatively. Therefore, the capsule should be preserved and utilized for capsular closure. We did not find any complication with the use of the technique.

The bone quality around the hip joint affects the stability of prosthesis. During reamerization, Cebesoy et al. [22] found poor bone quality at both the acetabulum and proximal femur irrespective of the patient's age. In our cases, we noticed the bone quality of the proximal femur was in accordance with the age of patients, but acetabulum wall was sclerotic, which was attributed to deposition of pigments in the deeper layers of articular cartilage (Fig. 4b). As a result, the cartilage loses its elasticity and become sclerotic [15]. In view of the matter, we advise to secure the cup with screws, because the sclerotic rim may impede the cup expansion.

Before 1980, we did cemented THR because we did not had other choices, and in the rest of the hips we used cementless THR. Because the bone tissue is uncommonly involved, we were not suspecting any bone-ingrowth deficits that might affect stability of the cementless implants $[15,23]$. In the present study, we didn't observe instability, early loosening, subsidence, or protrusion problems on radiographs. Radiolucent lines, migration, or change in alignment were not observed on the acetabular socket. These factors are suggestive of stable implant with bone growth (spot welding). On femoral side, we did not find subsidence, radiolucent lines, or instability of femoral components (Fig. 6a, b, c, d, e, f, g and Fig. 7a, b, c, d). Long-term follows showed
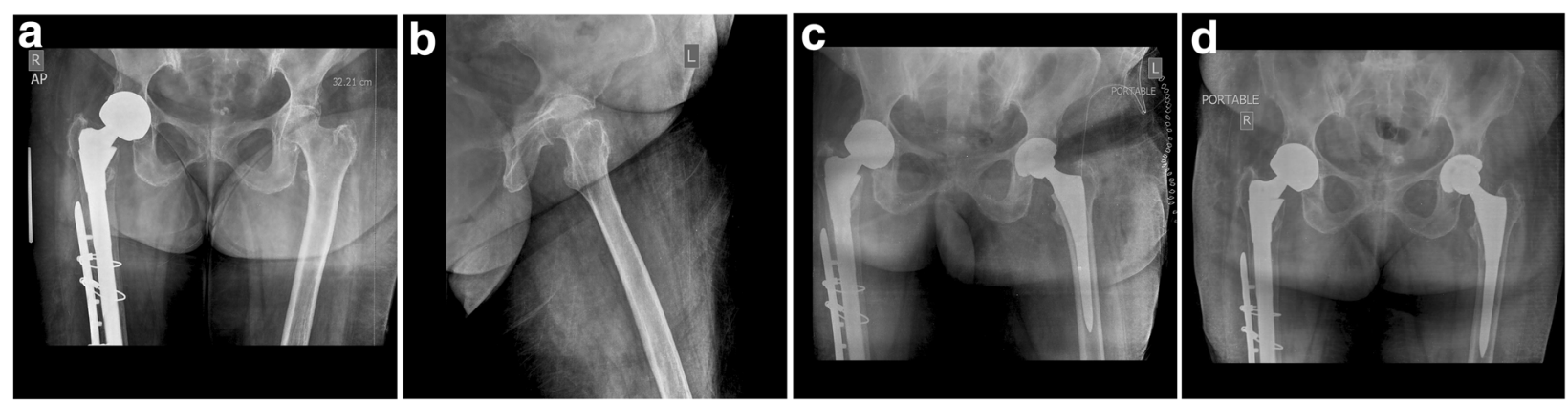

Fig. 7 Pelvis and hips on X-rays. a an anteroposterior view preoperatively (left); follow-up X-rays after 2 years (uncemented ASR with S-ROM; right); b preoperative lateral view of left hip; $\mathbf{c}$ an anteroposterior view immediately after surgery (left) and follow-up after 2 years (right); $\mathbf{d}$ anteroposterior view at the final follow-up of 15.7 years (Uncemented ASR with S-ROM; right) and left hip (13.7 years; Pinnacle with Summit) 
that superior pain relief and successful restoration of hip function were achieved (mean follow-up time: $16.70 \pm 6.82$ years; range, 3 to 24 years) in patients with ochronotic arthropathy. We therefore conclude that both the uncemented and cemented total hip had long term survivorship. Our preference is to use a cemented THR in patients with poor bone stock or osteoporotic bone. Furthermore, in view of patient's stiff spine in present series, we strongly believe that in the early era of total hip arthroplasty in India, very little or no significance was attributed to the stiffness of spine and /or deformity of spine. We have operated on the patients in present series for a long time before the inception of concept of spinopelvic mobility and total hip arthroplasty. The concept of spinopelvic parameters/movements in relation to THR is new. So, we did not have preoperative lateral spinopelvic-hip X-rays, nor sitting or standing lateral spine X-rays. In the early years, we had intraoperatively used manual jigs and eyeballing to achieve optimal component alignment with respect to patient's anatomy and limb length. Versions/offsets were attended independently to ensure optimal component positioning and a stable hip. In cemented hip, the principals of Charnley hip were followed to measure various angles. Recently, we used the principles of Scott Ranawat coplannar test in uncemented surgery. Fortunately, there was no dislocation in these groups of patients, in spite of a 22$\mathrm{mm}$ femoral head used.

There is no specific medical treatment for alkaptonuria, and hence all therapeutic approaches are symptomatic. In severe osteoarthrosis, total hip arthroplasty is the preferred treatment. Results from our series are consistent with the available literature [2, 22, 24-26]. The findings support reliability of THR in patients with ochronotic arthropathy. We hope the rationale behind the present series can guide the surgeons to establish the pre-operative diagnosis and to facilitate the intra-operative procedures. No complications related to implant failure were detected after total arthroplasty, and our results are compatible with patients who underwent primary osteoarthrosis.

\section{Conclusion}

Primary hip arthroplasty is an effective and preferred treatment for alkaptonuric hip arthritis. The critical factor that directly influences the outcomes of total arthroplasty is surgeons' acquaintance with the ochronosis. Through these cases we have attempted to provide tips pertinent to the establishment of the diagnosis of ochronosis and performance of THR for alkaptonuric hip arthritis. Those tips can help to lower surgical complications.

\section{Abbreviations}

DUS: Dried urine spot; HGA: Homogentisic acid; HHS: Harris hip score; THR: Total hip replacement

\section{Acknowledgements}

We acknowledge all the patients who participated in the study, nursing, paramedical staff. We also acknowledge the contribution of entire research team.

\section{Authors' contributions}

JAP (Conceptualization; Data curation; Investigation; Methodology; Supervision; Writing-review \& editing). VIS (Resources). SU

(Conceptualization; Data curation; Formal analysis; Investigation; Methodology; Resources; Supervision; Validation; Visualization; Writing review \& editing). KS (Resources). AS (Resources). AK (Resources). All authors read and approved the final manuscript.

\section{Funding}

Not applicable

\section{Availability of data and materials}

The data that support the findings of this study are available from [Shalby Hospitals India] but restrictions apply to the availability of these data, which were used under license for the current study, and so are not publicly available. Data are however available from the authors upon reasonable request and with permission of [Shalby Hospitals India].

\section{Ethics approval and consent to participate}

The study was approved by the Scientific Review Committee and the institutional review board of the participating Health Service. Written Informed consent (about the surgical technique, risks and potential complications) was provided according to the Declaration of Helsinki and obtained from all participating patients.

\section{Consent for publication}

Informed consent was obtained from the patient's for publication of their case records for providing evidence-based scientific literature for further research.

\section{Competing interests}

The authors declare that they have no competing interests.

\section{Author details}

'Department of Hip Arthroplasty, Shalby Hospitals, Ahmedabad, Gujarat, India. ${ }^{2}$ Department of Knee, Shalby Hospitals, Ahmedabad, India.

${ }^{3}$ Department of Orthopaedics, NSCB Medical College, Jabalpur, MP, India. ${ }^{4}$ Department of Trauma, Joint Replacement and Minimal Invasive Surgery, Shalby Hospitals Jabalpur, Jabalpur, Madhya Pradesh, India. ${ }^{5}$ Department of Knee and Hip Arthroplasty, Shalby Hospitals, Ahmedabad, Gujarat, India.

Received: 22 March 2019 Accepted: 12 August 2019

Published online: 03 October 2019

\section{References}

1. Keller JM, Macaulay W, Nercessian OA, Jaffe IA. New developments in ochronosis: review of the literature. Rheumatol Int. 2005;25:81-5.

2. Spencer JM, Gibbons CL, Sharp RJ, Carr AJ, Athanasou NA. Arthroplasty for ochronotic arthritis: no failure of 11 replacements in 3 patients followed 6-12 years. Acta Orthop Scand. 2004;75:355-8.

3. Collins EJ, Hand R. Alkaptonuric ochronosis: a case report. AANA J. 2005:73:41-6.

4. Borman P, Bodur H, Ciliz D. Ochronotic arthropathy. Rheumatol Int. 2002;21:205-9.

5. Sahin G, Milcan A, Bagis S, Kokturk A, Pata C, Erdogan C. A case of ochronosis: upper extremity involvement. Rheumatol Int. 2001;21:78-80.

6. Kumar RV, Rajasekaran S. Spontaneous tendon ruptures in alkaptonuria. J Bone Joint Surg (Br). 2003;85:883-6.

7. Fisher AA, Davis MW. Alkaptonuric ochronosis with aortic valve and joint replacements and femoral fracture: a case report and literature review. Clin Med Res. 2004;2:209-15.

8. Demir S. Alkaptonuric ochronosis: a case with multiple joint replacement arthroplasties. Clin Rheumatol. 2003;22:437.

9. Jacomelli G, Micheli V, Bernardini G, Millucci L, Santucci A. Quick diagnosis of alkaptonuria by homogentisic acid determination in urine paper spots. JIMD Rep. 2016;31:51-6. 
10. Janocha S, Wolz W, Srsen S, Srsnova K, Montagutelli X, Guenet JL, Grimm T, Kress W, Mueller CR. The human gene for alkaptonuria (AKU) maps to chromosome 3q. Genomics. 1994;19:5-8.

11. Nas K, Gür A, Akdeniz S, Cevik R, Harman M, Saraç AJ. Ochronosis: a case of severe ochronoticarthropathy. Clin Rheumatol. 2002;21:170-2.

12. Wauthy P, Seghers V, Mathonet P, Deuvaert FE. Cardiac ochronosis: not so benign. Eur J Cardiothorac Surg. 2009;35:732-3.

13. Kusakabe N, Tsuzuki N, Sonada M. Compression of the cervical cord due to alcaptonuric arthropathy of the atlanto-axial joint. A case report. J Bone Joint Surg Am. 1995;77:274-7.

14. Phornphutkul C, Introne WJ, Perry MB, Bernardini I, Murphey MD, Fitzpatrick DL, Anderson PD, Huizing M, Anikster Y, Gerber LH, Gahl WA. Natural history of alkaptonuria. N Engl J Med. 2002;347:2111-21.

15. Laskar FH, Sargison KD. Ochronotic arthropathy. A review with four case reports. J Bone Joint Surg. 1970;52:653-66.

16. Ranganath LR, Cox TF. Natural history of alkaptonuria revisited: analyses based on scoring systems. J Inherit Metab Dis. 2011;34:1141-51.

17. EISENBERG H, et al. Arch Intern Med. 1950;86:79.

18. Cervenansky J, Sitaj S, Urbanek T. Alkaptonuria and ochronosis. J Bone Joint Surg. 1959;41:1169.

19. Resnick D. Alkaptonuria. In: Resnick D, Niwayama G, editors. Diagnosis of bone and joint disorders. 2nd ed. Philadelphia: W. B. Saunders; 1988. p. 1787-803

20. O'Brien W, La DB, Bunim JJ. Biochemical, pathologic and clinical aspects of alcaptonuria, ochronosis, and chronotic arthropathy. Am J Med. 1963; 34:813-38.

21. Rose GK. Ochronosis. Br J Surg. 1957;44:481.

22. Cebesoy O, Isik M, Subasi M, Kaya A, Bilgin F, Kaya O. Total hip replacement for an ochronotic patient: a technical trick. Am J Case Rep. 2014;15:27-30.

23. Aydogdu S, Cullu E, Ozsoy MH, Sur H. Cementless total knee arthroplasty in ochronotic arthropathy: a case report with a 4-year follow-up. J Arthroplast. 2000;15:539-43.

24. Carrier DA, Harris CM. Bilateral hip and bilateral knee arthroplasties in a patient with ochronotic arthropathy. Orthop Rev. 1990;19:1005-9.

25. Araki K, Sudo A, Hasegawa M, Uchida A. Devastating ochronotic arthropathy with successful bilateral hip and knee arthroplasties. J Clin Rheumatol. 2009; 15:138-40.

26. Moslavac A, Moslavac S, Cop R. Case report of a patient with ochronosis and arthroplasty of the hip and both knees. Reumatizam. 2003;50:26-8.

\section{Publisher's Note}

Springer Nature remains neutral with regard to jurisdictional claims in published maps and institutional affiliations.

Ready to submit your research? Choose BMC and benefit from:

- fast, convenient online submission

- thorough peer review by experienced researchers in your field

- rapid publication on acceptance

- support for research data, including large and complex data types

- gold Open Access which fosters wider collaboration and increased citations

- maximum visibility for your research: over $100 \mathrm{M}$ website views per year

At $\mathrm{BMC}$, research is always in progress.

Learn more biomedcentral.com/submissions 